\title{
Author Correction: Sex Differences for Preferences of Shoulder to Hip Ratio in Men and Women: an Eye Tracking Study
}

\author{
Farid Pazhoohi ${ }^{1}$ - Ray Garza ${ }^{2}$ - James F. Doyle ${ }^{3}$. Antonio F. Macedo ${ }^{4,5}$ - Joana Arantes ${ }^{1}$ \\ Published online: 11 July 2019 \\ (C) Springer Nature Switzerland AG 2019
}

\section{Author Correction: Evolutionary Psychological Science https://doi.org/10.1007/s40806-019-00198-w}

The original version of this article contained mistakes. In Abstract section, "Also, no differences were observed for female participants in dwell time, for either chest regions of SHRs of male stimuli or for the chests of female stimuli", is incorrect and "Also, differences were observed for female participants in dwell time for the chest region (i.e., upper back) of back posed male stimuli" is correct. In the Eye Tracking subsection of Results, "a 3(SHR: Low, Intermediate, High) by 3(ROI: Head, Chest, Waist, Hips/thighs, Legs/Feet) repeated measures ANOVA", is incorrect and "A 3(SHR: Low, Intermediate, High) by 5(ROI: Head, Chest, Waist, Hips/thighs, Legs/Feet) repeated measures ANOVA" is correct. In Discussion section, "Also, no differences were observed for female participants in dwell time, for either chest region of SHRs of male stimuli or for the chest of female stimuli (Fig. 4). Consistently, recent research has found no differences for attentional biases to regions of interest as a function of the waist to chest ratios (Garza et al. 2017; Garza and Byrd-Craven 2019)" is incorrect, and "Similarly, female

The online version of the original article can be found at https://doi.org/ 10.1007/s40806-019-00198-w

Farid Pazhoohi

pazhoohi@gmail.com

1 Department of Basic Psychology, School of Psychology, University of Minho, Braga, Portugal

2 Department of Psychology, Oklahoma State University, Stillwater, OK, USA

3 Stillwater, MN, USA

4 Vision Rehabilitation Laboratory, Department and Centre of Physics, University of Minho, Braga, Portugal

5 Department of Medicine and Optometry, Linnaeus University, Kalmar, Sweden participants had longer dwell times for the chest region (i.e., upper back) of back posed male stimuli, but not front posed male stimuli (Fig. 4). Consistently, recent research has found no differences in women for attentional biases to regions of interest as a function of the waist to chest ratios in front facing male stimuli (Garza et al. 2017; Garza and Byrd-Craven 2019)" is correct.

Figures 1, 2, 3, 4, 5, 6, 9 and 10 are displaying the results for 4- and 5-way ANOVAs reported below, while only twoway significant interactions are reported in the paper.

\section{Results}

\section{Ratings of Attractiveness}

The interrater reliability for male participants was high. The average measure interclass correlation coefficient for male participants was .876 with a $95 \%$ confidence interval from .747 to $.957(F(11,341)=8.041, p<.001)$. Similarly, for female participants average measure ICC was high: 0.968 with a 95\% confidence interval from .935 to $.989(F(11,539)=$ $30.927, \mathrm{p}<.001)$.

A 2 (Participant Sex $) \times 2$ (Stimulus Sex $) \times 2$ (Side: Front and Back views $) \times 3$ (SHR: low, intermediate and high) mixed ANOVA was performed with Participant Sex as a betweensubjects variables and Stimulus Sex, Side, and SHR as withinsubjects variables. All post hoc comparisons were done using Bonferroni correction. The main effects for stimulus sex, view side and SHR were significant (stimulus sex: $F(1,80)=13.69$, $p<.001, \eta^{2}=0.14$; view side: $F(1,80)=4.14, p=.045, \eta^{2}=$ 0.05 ; SHR: $\left.F(2,160)=12.63, p<.001, \eta^{2}=0.13\right)$. Significant two-way and three-way interactions were qualified by a significant four-way Stimulus Sex $\times$ Side $\times$ SHR $\times$ Participant Sex interaction, $F(2,160)=6.88, p=.001, \eta^{2}=0.08$. 
Figure 1 shows the male participants' ratings for physical attractiveness of male and female stimuli as a function of SHR and side view. When viewing male stimuli in front view, male participants rated the high SHR $(\mathrm{M}=4.97, \mathrm{SE}=0.34)$ significantly higher than both intermediate $(\mathrm{M}=4.28, \mathrm{SEM}=$ $0.28, p<.001)$ and low SHR $(\mathrm{M}=3.93, \mathrm{SEM}=0.25$, $p<.001)$. They also rated the attractiveness of intermediate SHR higher than low SHR $(t=-2.57, d f=31, p=.005$; see Fig. 1a). Male participants viewing the male back stimuli rated high SHR $(M=6.17, \mathrm{SEM}=0.34)$ significantly higher than both intermediate $(\mathrm{M}=6.06, \mathrm{SEM}=0.34, p<.001)$ and low SHR $(\mathrm{M}=5.03, \mathrm{SEM}=0.30, p<.001)$; and rated intermediate SHR higher than low SHR $(p<.001$; see Fig. 1a).

Male participants viewing the female back stimuli rated intermediate SHR $(M=5.91, \mathrm{SEM}=0.28)$ significantly higher than low $(\mathrm{M}=5.46, \mathrm{SEM}=0.30, p=.004)$ and high SHR $(\mathrm{M}=5.67, \mathrm{SEM}=0.30, p=.008$; see Fig. 1b). Additionally, male participants rated high SHR $(\mathrm{M}=4.26, \mathrm{SEM}=0.33)$ lower than both low $(\mathrm{M}=5.18, \mathrm{SEM}=0.29, p<.001)$ and intermediate SHR ( $\mathrm{M}=4.99$, SEM $=0.32, p<.001)$ of female stimuli in back view. No difference was observed between low and intermediate SHR ( $p=.120$; see Fig. 1b).

Figure 2 shows the female participants' ratings for the physical attractiveness of male and female stimuli as a function of SHR and view side. No difference in female participants' ratings of the attractiveness of male stimuli in front view was observed between the SHR (all $p s>.555$; see Fig. 2a). For back view of male stimuli, female participants rated the intermediate SHR $(M=5.40, S E M=0.27)$ significantly higher than both high $(\mathrm{M}=5.02, \mathrm{SEM}=0.29, p=.010)$ and low SHR $(\mathrm{M}=$ $4.90, \mathrm{SEM}=0.24, p<.001)$. No difference was found between high and low SHRs ( $p=.469$; see Fig. 2a).

No significant difference was observed when female participants viewed female stimuli in front view (all $p s>.072$; see Fig. 2b). When female participants viewed back female stimuli, intermediate SHRs $(M=3.92, \mathrm{SEM}=0.26)$ were rated significantly higher than high SHR $(\mathrm{M}=3.70, \mathrm{SEM}=0.27$, $p=.008$ ). No other difference was significant ( $p s>.169$; see Fig. 2b).

\section{Eye-Tracking}

Dwell Time For dwell time, a 2 (Participant Sex) $\times 2$ (Stimulus Sex $) \times 2$ (Side: Front and Back views) $\times 3$ (SHR: low, intermediate and high) $\times 5$ (ROI) mixed ANOVA was performed, with Participant Sex as a between-subjects variables and Stimulus Sex, Side, SHR, and ROI as within-subjects variables. The ANOVA revealed a significant main effect for ROI, $F(4,320)=44.65, p<.001, \eta^{2}=0.35$. Significant twoway and three-way interactions including ROI were qualified by significant four-way Stimulus Sex $\times$ SHR $\times$ ROI $\times$ Participant Sex, $F(8,640)=4.85, p<.001, \eta^{2}=0.05$, and Stimulus Sex $\times$ Side $\times$ ROI $\times$ Participant Sex interactions,
$F(4,320)=4.59, p=.001, \eta^{2}=0.05$. Figures 3 and 4 show dwell times for male and female participants on different body regions of male and female stimuli as a function of SHR. Stimulus Sex $\times$ SHR $\times$ ROI $\times$ Participant Sex interaction showed that when looking at male stimuli, men had higher dwell time on high SHR (M=1677.75 milliseconds, SEM = 132.56) compared to intermediate $(\mathrm{M}=1303.12$, $\mathrm{SEM}=$ 114.25.54, $p<.001)$ and low SHRs $(\mathrm{M}=906.42, \mathrm{SEM}=$ 91.54, $p<.001$; Fig. 3). Men looked more at intermediate SHR compared to low SHR $(p<.001)$. No other difference was observed regarding SHR, for either male or female participants (see Figs. 3 and 4).

Figures 5 and 6 show dwell time for male and female participants made on different body regions of male and female stimuli as a function of viewed side. Stimulus Sex $\times$ Side $\times$ ROI $\times$ Participant Sex interaction indicated that both men and women had longer dwell time on the upper back of male stimuli (men: $\mathrm{M}=1006.24$ milliseconds, $\mathrm{SEM}=119.56$; women: $\mathrm{M}=949.85, \mathrm{SEM}=95.65)$ compared to their chest (men: $\mathrm{M}=1585.29, \mathrm{SEM}=108.48, p<.001$; women: $\mathrm{M}=$ 1199.85, SEM $=86.78, p<.001)$. See Figs. 7 and 8 for an example of heat maps on male and female stimuli for all the participants.

Number of Fixations For number of fixations, a 2 (Participant Sex $) \times 2($ Stimulus Sex $) \times 2($ Side: Front and Back views $) \times 3$ (SHR: low, intermediate and high) $\times 5$ (ROI) repeated measures ANOVA was performed, with Participant Sex as a between-subjects variables and Stimulus Sex, Side, SHR, and ROI as within-subjects variables. The ANOVA revealed a significant main effect for ROI, $F(4,320)=66.67, p<.001$, $\eta^{2}=0.45$. Significant two-way and three-way interactions including ROI were qualified by a significant four-way Stimulus Sex $\times$ Side $\times$ ROI $\times$ Participant Sex interaction, $F(4,320)=$ $3.00, p=.018, \eta^{2}=0.36$. Figures 9 and 10 show dwell time for male and female participants on different body regions of male and female stimuli as a function of view side. This interaction indicated that both men and women had higher fixations on upper back of male stimuli (men: $\mathrm{M}=3.93$, $\mathrm{SEM}=0.25$; women: $\mathrm{M}=3.57, \mathrm{SEM}=0.20$ ) compared to their chest (men: $\mathrm{M}=2.89, \mathrm{SEM}=0.23, p<.001$; women: $\mathrm{M}=2.70$, $\mathrm{SEM}=0.19, p<.001)$. Also, men fixated more on the upper back of female stimuli $(\mathrm{M}=3.28, \mathrm{SEM}=0.22)$ than their chest $(\mathrm{M}=2.68, \mathrm{SEM}=0.21, p=.016$; Figs. 9 and 10 ; see Tables S1 and S2 for correlations between attractiveness ratings and dwell time and fixation numbers for male and female participants).

The authors apologize for this oversight and for any confusion it may have caused.

Publisher's Note Springer Nature remains neutral with regard to jurisdictional claims in published maps and institutional affiliations. 